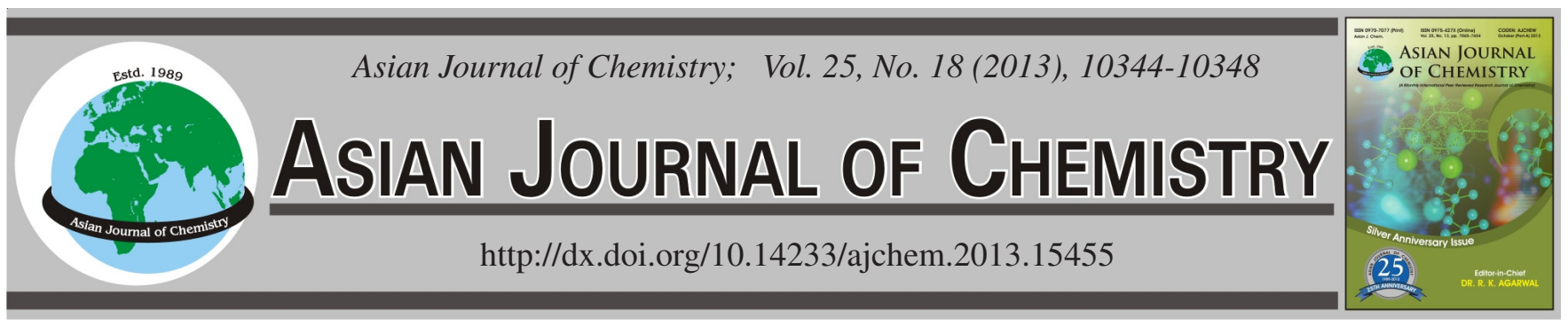

\title{
Quantification of Ten Polyphenols in the Leaves of Chinese Hawthorn (Crataegus pinnatifida Bge. var. major N.E. Br.) by HPLC
}

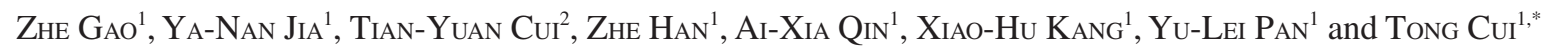

${ }^{1}$ College of Food Science and Technology, Agricultural University of Hebei, Baoding 071001, Hebei Province, P.R. China ${ }^{2}$ College of Pharmaceutical Science, Hebei University, 180 Wusidong Road, Baoding City, Hebei Province, P.R. China

*Corresponding author: Fax: +86 312 7528195; Tel: +86 312 7528427; E-mail: cuitong98@yahoo.com.cn

\begin{abstract}
The variation of the contents of vitexin-2"-rhamnoside, vitexin-glucoside, vitexin, hyperoside, isoquercitrin, chlorogenic acid, eucomic acid, epicatechin, procyanidin B2 and $\mathrm{C} 1$ in leaves of Chinese hawthorn (Crateagus pinnatifida Bge. var. major N.E.Br.) were evaluated by a HPLC method. The total content of the phenols in young leaves was $11.90 \mathrm{mg} / \mathrm{g}$ dry weight and the main constituents were vitexin$2^{\prime \prime}$-rhamnoside $(5.22 \mathrm{mg} / \mathrm{g}$ dry weight $)$ and vitexin-glucoside $(3.50 \mathrm{mg} / \mathrm{g}$ dry weight). Eucomic acid was particularly found in Chinese hawthorn, its content was around $2.5 \mathrm{mg} / \mathrm{g}$ dry weight. The contents of the other 7 phenols increased gradually during the growth period. On October 7th, the total content of the ten phenols reached the highest level (21.49 $\mathrm{mg} / \mathrm{g}$ dry weight), in which the total amount of the 5 flavonoids was $11.57 \mathrm{mg} / \mathrm{g}$ dry weight and that of the three procyanidins was $5.35 \mathrm{mg} / \mathrm{g}$ dry weight and the chlorogenic acid was $2.03 \mathrm{mg} /$ $\mathrm{g}$ dry weight. At that time, the differences between total contents of phenolic in leaves on different parts of twigs were not significant. But the rate of the $\mathrm{C}$-glycosideflavonoid/O-glycosideflavonoid decreased from 8.08 in the first leaves to 1.83 in the 8 th leaves. The style of main polyphenols in young leaves of Chinese hawthorn was different from the European hawthorn $($ C. oxyacantha).
\end{abstract}

Key Words: Hawthorn, Crataegus pinnatifida, Polyphenols, Flavonoids, Eucomic acid, Procyanidins, HPLC.

\section{INTRODUCTION}

In Europe, the standard extracts of the leaves and flowers of hawthorn (Crataegus oxyacantha or Crataegus monogyna) have been used for treating mild congestive heart failure $(\text { NYHA II })^{1}$. Flavonoids and procyanidins were considered as the most important active components in them ${ }^{2}$. These components in European hawthorn leaves have been analyzed by HPLC method ${ }^{3-5}$.

In China, the extracts of leaves of Chinese hawthorn have also been developed into preparations and have been included in Chinese Pharmacopoeia ${ }^{6}$. Hu et al. ${ }^{7}$ have analyzed the content of vitexin in dried leaves of $C$. pinnatifida by HPLC. Li et al. ${ }^{8}$ determined vitexin-2"-rhamnoside of Chinese hawthorn leaves at different growth time. Liu et al. ${ }^{9}$ compared the concentrations of seven flavonoids and chlorogenic acid in dried leaves of Crataegus L from eight province of China. Besides, the content of procyanidins, another group of important polyphenols in Chinese hawthorn leaves, has not been reported. Recently, we isolated a compound with typical fragrance from Chinese hawthorn fruits and confirmed it is eucomic acid; which is a kind of secondary metabolite rarely seen in plants and is mentioned in previous papers ${ }^{10-14}$. Ding et al. ${ }^{12}$ reported its existence in leaves of $C$. pinnatifida. According to the research on Vanda teres, eucomic acid contributed to stimulate respiratory functions in keratinocytes by increasing cytochrome $c$ oxidase activity and/or expression and is therefore a potential new natural ingredient for antiaging preparations to remedy age-related disorders. but its level in leaves of Chinese or European hawthorn has not been reported yet. Recently, we systematically analyzed the flavonoids, phenolic acids and procyanidins in Chinese hawthorn fruits by HPLC ${ }^{15-17}$. In present research, flavonoids, procyanidins and phenolic acids (Fig. 1) in hawthorn leaves in different growth periods and different leaf sequences were analyzed to evaluate the level of the phenol compounds.

\section{EXPERIMENTAL}

Chlorogenic acid (CA), epicatechin (Epi) and rutin (Ru) were purchased from Sigma Chemical Co. (St. Louis, MO, USA); procyanidin B2 (B2), vitexin (V), vitexin-2"-rhamnoside, (VR), hyperoside, (Hy) and isoquercitrin (IQ), were purchased from Funakoshi Co. Ltd. (Tokyo, Japan); acetonitrile and water used for the HPLC mobile phase were HPLC grade. Procyanidins C1, D1 and vitexin-glucoside (VG) were isolated and purified from the fruits of $C$. pinnatifida Bge. var. major 
<smiles>Oc1cc(O)c2c(c1)O[C@H](c1ccc(O)c(O)c1)[C@H](O)C2</smiles>

Epicatechin<smiles>[R]c1c(-c2ccc(O)c(O)c2)oc2cc(O)cc(O)c2c1=O</smiles>

hyperoside $(\mathrm{R}=$ galactoside $)$ isoquercitrin $(\mathrm{R}=$ glucoside $)$

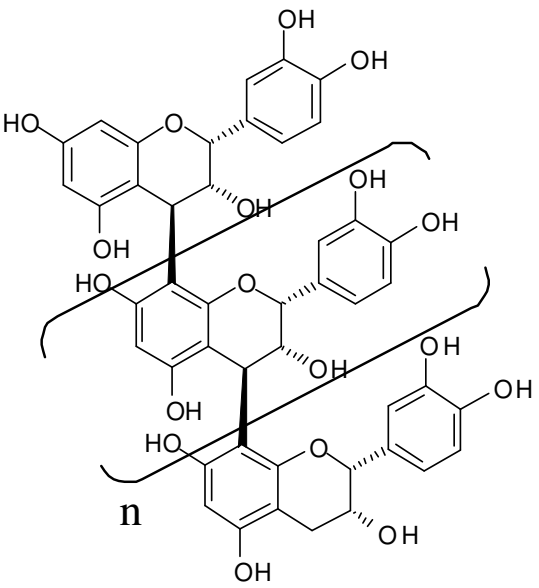

procyanidin $\mathrm{B} 2(\mathrm{n}=0)$ procyanidin $\mathrm{C} 1(\mathrm{n}=1)$ procyanidin D1 $(\mathrm{n}=2)$

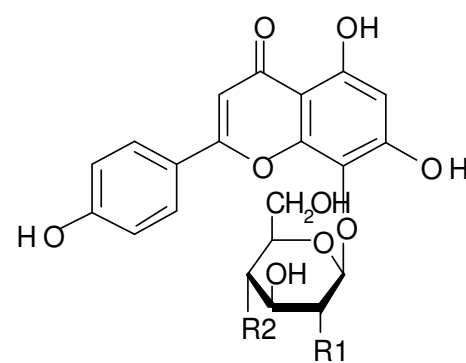

Vitexin $(\mathrm{R} 1=\mathrm{R} 2=\mathrm{OH})$

vitexin-2"-rhamnoside $(\mathrm{R} 1=$ rhamnose $\mathrm{R} 2=\mathrm{OH})$ vitexin-glucoside $(\mathrm{R} 1=\mathrm{OH} \mathrm{R} 2=$ glucose $)$<smiles>CCC(O)(CCCC(=O)C=CCCCO)C(=O)O</smiles>

Eucomic acid

Chlorogenic acid

Fig. 1. Polyphenols in Leaves of Chinese Hawthorn (Crataegus pinnatifida Bge. var. major $\mathrm{NE} \mathrm{Br}$ )

N.E. Br. and were identified by electrospray ionization-mass spectrometry (ESI-MS) and the thiolysis method ${ }^{16,17}$. The vitexin-glucoside used for peak identification was isolated from the leaves of $C$. pinnatifida Bge. var. major N.E. Br. and confirmed by MS and photometric methods ${ }^{17}$. Eucomic acid was isolated and confirmed according to the method below:

One kg (FW) fruits of Crateagus pinnatifida Bge. var. major N.E.Br. were cut in pieces and extracted three times with $1.5 \mathrm{~L}$ of $95 \%$ ethanol. Ethanol extracts were mixed

together and evaporated to $500 \mathrm{~mL}$ at $40{ }^{\circ} \mathrm{C}$ and extracted with $300 \mathrm{~mL}$ of petroleum ether three times to remove the lipid components and were then extracted with $500 \mathrm{~mL}$ of ethyl acetate for three times. The ethyl acetate layer was evaporated to produce a final extract of $6 \mathrm{~g}$. The extract was dissolved in $30 \mathrm{~mL}$ water and $15 \mathrm{~mL}$ this solution was added to a Sephadex LH 20 column (300 $\mathrm{mm} \times 40 \mathrm{~mm}$ i.d.) and eluted with methanol in water at 5, 10, 40, 60, 80 and $100 \%(\mathrm{v} / \mathrm{v}$, each $800 \mathrm{~mL}$ ), respectively. Each $200 \mathrm{~mL}$ eluate was collected and monitored by HPLC. The eluates from 3rd to 5th fractions were mixed, evaporated (under reduced pressure) and lyophilized to obtain $574 \mathrm{mg}$ of fraction A. The fraction A was dissolved in $30 \%(\mathrm{v} / \mathrm{v})$ methanol and were injected into a preparative HPLC system equipped with a Cosmosil $5 \mathrm{C}_{18^{-}}$ AR-300 column $(250 \mathrm{~mm} \times 20 \mathrm{~mm}$ i. d., $5 \mu \mathrm{m}$, Nacalai Tesque Inc., Kioto, Japan). The elution was carried out with $23 \%$ methanol $(\mathrm{v} / \mathrm{v})$ in $0.02 \%$ formic acid and the flow rate was eight $\mathrm{mL} / \mathrm{min}$. The chromatography was repeated and the fractions with retention time from 17 to $18 \mathrm{~min}$ were collected and lyophilized to obtain $127 \mathrm{mg}$ of compound $\mathbf{1}$. By electrospray-ionization mass spectrometry analysis, compound $\mathbf{1}$ generated the MS peaks with $\mathrm{m} / \mathrm{z}$ as $263\left[(\mathrm{M}+\mathrm{Na})^{+}\right]$. The ${ }^{1} \mathrm{H}$, ${ }^{13} \mathrm{C}$ and $2 \mathrm{D}$ NMR analysis were carried out by a Bruker DRX500 spectrometer (Bruker BioSpin Crop., Billerica, MA). NMR $\delta_{\mathrm{H}}\left(\mathrm{CD}_{3} \mathrm{SO}_{2} \mathrm{CD}_{3}\right): 6.981(2 \mathrm{H}, \mathrm{d}, J=8 \mathrm{~Hz}, \mathrm{H}-2$ ', H-6'), $6.62\left(2 \mathrm{H}, \mathrm{d}, J=8 \mathrm{~Hz}, \mathrm{H}-3\right.$ ', H-5'), 6.62 (2H, d, $J=8 \mathrm{~Hz}, \mathrm{H}-3^{\prime}$, H-5'), 2.795 (1H, d, $J=13.5 \mathrm{~Hz}, 7^{\prime}$ b), 2.741 (1H, d, $J=13.5$ Hz, 7'a), 2.692 (1H, d, $J=16 \mathrm{~Hz}, 3 \mathrm{~b}), 2.347$ (1H, d, $J=16$ $\mathrm{Hz}, 3 \mathrm{a}) . \mathrm{NMR} \delta_{\mathrm{C}}\left(\mathrm{CD}_{3} \mathrm{SO}_{2} \mathrm{CD}_{3}\right): 177.8(\mathrm{C}-4), 174.4(\mathrm{C}-1)$, 154.7 (C-4'), 131.8 (C-2', C-6'), 127.1 (C-1'), 115.3 (C-3', C$\left.5^{\prime}\right), 76.3$ (C-2), 44.0 (C-7'), 43.1 (C-3). These data and the 2D NMR analysis, including ${ }^{1} \mathrm{H}-{ }^{1} \mathrm{H}$ COSY, HMQC, HMBC and NOESY spectra, were in accordance with those of reported in the literatures ${ }^{13,14}$. Therefore, compound $\mathbf{1}$ should be eucomic acid.

Hawthorn (C. pinnatifida Bge. var. major N.E. Br.) leaves were collected from the experimental farm of Hebei Agricultural University in Baoding (Hebei Province, China) from May 20th to October 7th (2011) at 4 weeks intervals. Collect ten twigs in each period and collect eight leaves from the base of a twig in order and then the ten pieces of leaves of the same leaf sequence were combined to a sample group and then dry in vacuum to constant weight at room temperature. European hawthorn (Crataegus oxyacantha) was cultivated in Mizukuti farm in Takamori Mati of Nagano Ken, Japan. The leaves were collected in May 10th (2006) and were lyophilized and preserved in $-20{ }^{\circ} \mathrm{C}$ till analysis.

Preparation of sample solutions: For the comparison of phenolic components in different growth period, the eight groups of leaves of every period were cut into two identical parts longitudinally and one part of the two was crushed into powder. Each $1 \mathrm{~g}$ of the powder sample was mashed into slurry with $5 \mathrm{~mL}$ of cold $\left(0^{\circ} \mathrm{C}\right)$ extraction solvent $(70 \%$ methanol in $0.1 \%$ of phosphoric acid) in a cold mortar at $0{ }^{\circ} \mathrm{C}$. The cream slurry was transferred into a $25 \mathrm{~mL}$ volumetric flask using $20 \mathrm{~mL}$ extraction solvent and was subjected to extraction in a US C-202 model ultrasonic bath (Polong Co., Shanghai, China) for $5 \mathrm{~min}$ before being brought to the volume using extraction solvent. After the supernatant was filtered through a $0.22 \mu \mathrm{m}$ 
membrane, the filtrate was injected for the HPLC determination. For the comparison of phenolic components in different leaf sequences, eight leave groups of the other half of the leaves collected on October 7th were crushed into powder respectively. Each $0.2 \mathrm{~g}$ of the eight powder samples was mashed and prepared followed the procedure described above. The sample of European hawthorn was also prepared followed the procedure described above. All of the samples were prepared in triplicate.

HPLC analysis: The chromatograph (Elite Analytical Instruments Co., Dalian, China) was equipped with two P200II solvent delivery units, a UV200 detector, an Elite system controller, an Echrom 98 chromatography workstation and a Hypersil BDS $\mathrm{C}_{18}(5 \mu \mathrm{m} ; 250 \mathrm{~mm} \times 4.6 \mathrm{~mm}$, i.d. $)$ column. The gradient elution was performed by using $50 \%(\mathrm{v} / \mathrm{v})$ of acetonitrile in $0.05 \%$ of formic acid as mobile phase $\mathrm{A}$ and $10 \%(\mathrm{v} / \mathrm{v})$ of acetonitrile in $0.05 \%$ of formic acid as mobile phase $\mathrm{B}$. The gradient procedure was $8 \% \mathrm{~A}$ at $0 \mathrm{~min} 43 \% \mathrm{~A}$ at $20 \min 100 \% \mathrm{~A}$ at $23 \min 100 \% \mathrm{~A}$ at $30 \min 8 \% \mathrm{~A}$ at 33 min and $8 \% \mathrm{~A}$ at $40 \mathrm{~min}$. The column temperature was $21^{\circ} \mathrm{C}$ and the wavelength used for detection was $280 \mathrm{~nm}$ for the procyanidins and phenolic acids analysis and $350 \mathrm{~nm}$ for the flavonoids analysis. The flow rate was $0.8 \mathrm{~mL} / \mathrm{min}$ and the injection volume was $10 \mu \mathrm{L}$. Stock solutions for the determination of the phenols were prepared at a concentration of 2 $\mathrm{mg} / \mathrm{mL}$ in methanol. Working solutions $(0-200 \mu \mathrm{g} / \mathrm{mL})$ were prepared by diluting the standard solutions with purified water.

\section{RESULTS AND DISCUSSION}

HPLC chromatogram: Fig. 2 is a HPLC profile of the phenolic constituents in leaves of Chinese hawthorn. It demonstrates that under this analysis condition, the five main flavonoids (VG, VR, V, Hy and IQ), four main procyanidins (Epi, B2, C1 and D1) and the two phenolic acid (CA and eucomic acid) have been well separated and each sample needs only $40 \mathrm{~min}$. According to the previous report ${ }^{18}$, we also analyzed rutin in the samples and the retention time of the reference was $19.2 \mathrm{~min}$. However, the results showed that the rutin content in hawthorn leaves was very low. Besides, Fig. 2(C) showed that the autumn leaves contain considerable procyanidin D1 (peak 6), but it was not analyzed in this experiment for the lack of enough amount of reference standard.

Fig. 2(A) is a chromatogram of samples collected on May 20th. It can be seen that except for VG and VR, the contents of the other constituents were lower than those in autumn leaves [Fig. 2 (B) and (C)] obviously.

Fig. 3 shows the comparison of HPLC profile between Chinese and European hawthorn leaves. (A) and (B) were determined at $280 \mathrm{~nm}$ and (C) and (D) were determined at $350 \mathrm{~nm}$. As the counterpart on Fig. 3(A), the European hawthorn leaves in the period contained high amount of CA. There is a big peak in the position of Epi peak, but it was not Epi. That can be seen more clearly in Fig. 3(C) and (D). These two profiles of young leaves of European hawthorn and Chinese hawthorn were detected at $350 \mathrm{~nm}$. The components of the flavonoids in Chinese hawthorn were relatively simple: the main components were VG and VR. However, the components in European hawthorn were relatively complicated and the

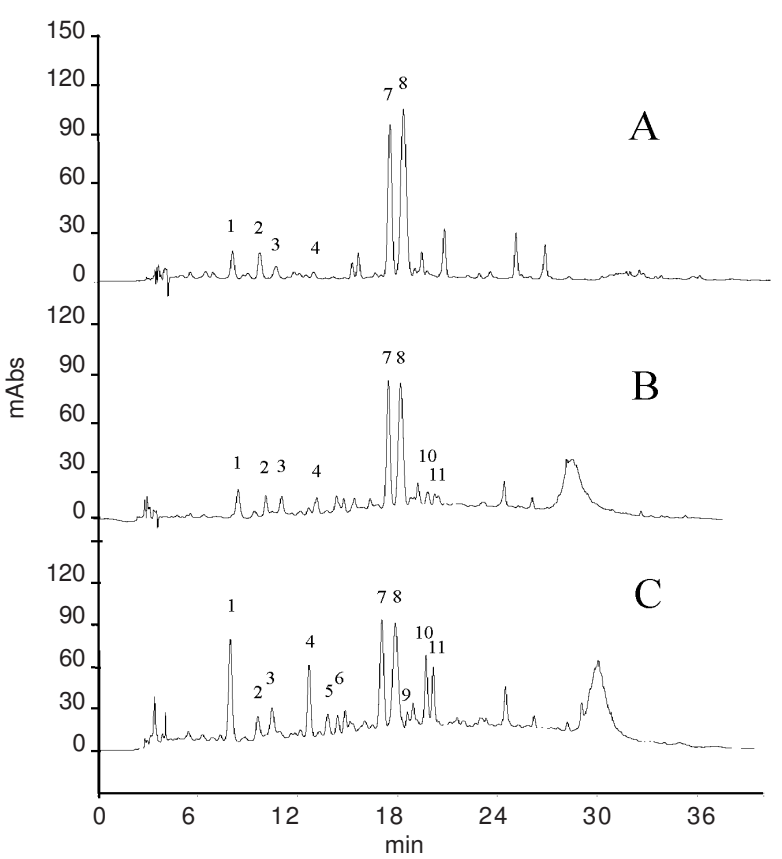

Fig. 2. HPLC Profile of Polyphenols in Leaves of Chinese Hawthorn (Crataegus. pinnatifida Bge. var. major NE Br). Determined at 280 nm, A: Young leaves (May 20th), B: Mid-stage leaves (July 15th) and $\mathrm{C}$ : Old leaves (October 7th). Peak numbers: 1: chlorogenic acid, 2: eucomic acid, 3: procyanidin B2, 4: epicatechin, 5: procyanidin $\mathrm{C} 1,6$ : procyanidin $\mathrm{D} 1,7$ : vitexin-glucoside, 8: vitexin2"-rhamnoside, 9: vitexin, 10: hyperoside, 11: isoquercitrin
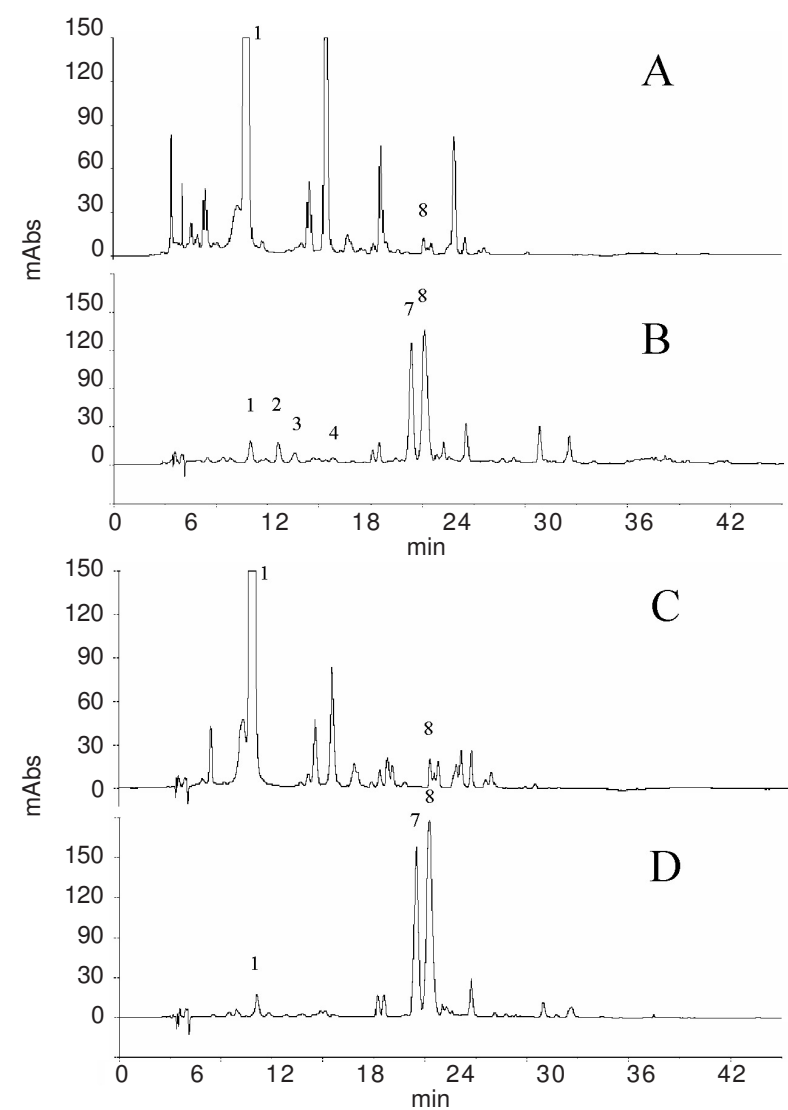

Fig. 3. Comparation of HPLC Profile Between Chinese and European Hawthorn Leaves. A: European hawthorn determined at $280 \mathrm{~nm}$, B: Chinese hawthorn determined at $280 \mathrm{~nm}, \mathrm{C}$ : European hawthorn determined at $350 \mathrm{~nm}, \mathrm{D}$ : Chinese hawthorn determined at $350 \mathrm{~nm}$. Peak numbers: 1: chlorogenic acid, 2: eucomic acid, 3: procyanidin B2, 4: epicatechin, 7: vitexin-glucoside, 8: vitexin-2"-rhamnoside 
retention time of chromatography peaks were not consistent with the references in this experiment. Another important characteristic of Chinese hawthorn was that it contained eucomic acid, the peak 2 in Fig. 3(B). However it was not seen in European hawthorn. This reviews the difference in phenolic components in different species of Crataegus.

Comparison in different growth periods: Fig. 4 shows the contents of ten main phenols in different growth periods and the data were shown in Table-1. The two C-glycosylflavones VR and VG are the main flavonoids in Chinese hawthorn leaves. This result was in accordance with previous reports ${ }^{19}$. During the growth period of the leaves, the contents of the two flavonoids decreased slightly. The content of vanadium, another kind of C-glycosylflavones, kept in a low level all the time and it was a kind of subordinary component. Another two O-glycosylflavones Hy and IQ were undetectable in young leaves, but gradually increase during the growth period of the leaves, especially in the autumn. At that time, the total content of the five flavonoids reached $11.56 \mathrm{mg} / \mathrm{g}$ dry weight. This level was close to the contents in European hawthorn ${ }^{20}$ and was obviously higher than the level in fruit of Chinese hawthorn ${ }^{16}$.

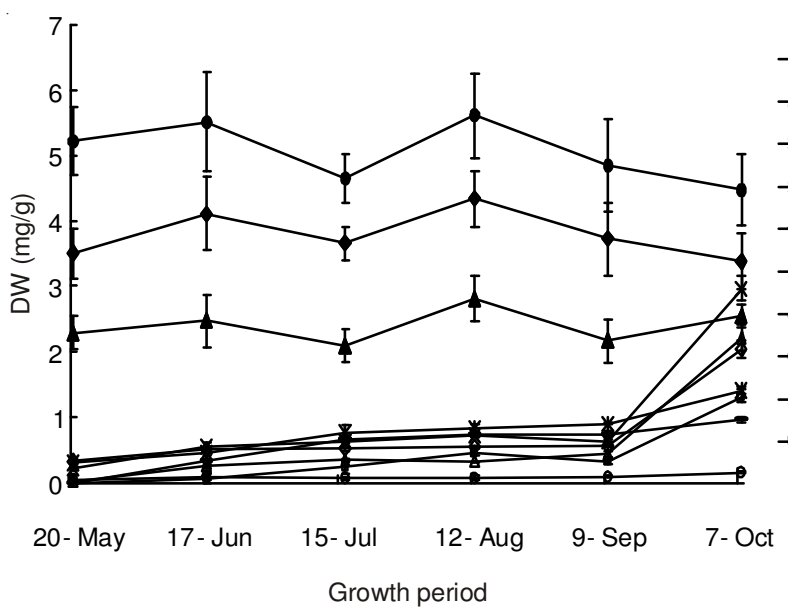

Fig. 4. Changes in Content of polyphenols in Leaves of Chinese Hawthorn (Crataegus. pinnatifida Bge. var. major NE Br) During Development. CA: chlorogenic acid, EA: eucomic acid, B2: procyanidin $\mathrm{B} 2, \mathrm{C} 1$ : procyanidin $\mathrm{C}$ 1, Epi: epicatechin, $\mathrm{VG}$ : vitexin-glucoside, VR: vitexin-2"-rhamnoside, V: vitexin, Hy: hyperoside, IQ: isoquercitrin. Values are expressed as means \pm SD of triplication
Another representative group of phenols in hawthorn leaves was procyanidins. From Fig. 2(A) it can be seen that their contents in young leaves were too low to make any sense. As the leaves grow, these constituents began to accumulate and then kept a relatively stable level in the middle period of growth. And then, these procyanidins obviously increased in autumn and reached their highest level in the last period. The total amount of the three procyanidin (Eip, B2 and C1) was $5.35 \mathrm{mg} / \mathrm{g}$ dry weight, which was slightly higher than the procyanidin level in fresh fruits of Chinese hawthorn ${ }^{16}$. The contents of these three procyanidins decreased according to the degree of polymerization (from monomer to trimer) and the mean degree of polymerization (mDP) of the oligomeric procyanidins (OPC) was 1.33, which was similar to the level in fruits of Chinese hawthorn ${ }^{16,17}$ and was lower than that of leaves of European hawthorn ${ }^{3}$.

Another group of phenols in hawthorn was phenolic acid. We have seen information concerning the content of chlorogenic acid (CA) in previous reports. Our results show that the content of CA in young leaves was not very high $(0.33 \mathrm{mg} / \mathrm{g}$ dry weight), but it reached up to $2.03 \mathrm{mg} / \mathrm{g}$ dry weight in the later part of the growth period. Fig. 2(A-C) and Table-1 showed that in leaves of Chinese hawthorn containing eucomic acid although the peak was not very high since its UV absorption was not very strong, its content was around $2.5 \mathrm{mg} / \mathrm{g}$ dry weight during the whole growth periods (Fig. 4) and was next below the two C-glycosylflavones. For these reasons eucomic acid should be an important secondary metabolite in Chinese hawthorns. Mun'im et al. ${ }^{14}$ has reported that this compound has weak antioxidant activity. Its other biological activities need further examination.

The total amount of the ten phenols in autumn leaves was $21.49 \mathrm{mg} / \mathrm{g}$ dry weight and it was obviously higher than that in other growth periods. This result was in accordance with the report (levels of quercetin and hyperoside) by Shi et al. ${ }^{21}$, but was different to another report Li et al. ${ }^{8}$. Shi et al. ${ }^{21}$ analyzed the variation of quercetin and hyperoside in hawthorn leaves in their growth period and found that the two O-glycosylflavones reached their highest level in autumn. Li et al. ${ }^{8}$ analyzed the variation of VR and total flavonoids in growth period and found that they had their highest level in August. We analyzed the ten kinds of charicristic components in Chinese hawthorn leaves and the total amount of these ten kinds of components reached its maximum of $21.49 \mathrm{mg} / \mathrm{g}$ dry weight in autumn.

\begin{tabular}{|c|c|c|c|c|c|c|}
\hline \multicolumn{7}{|c|}{$\begin{array}{l}\text { TABLE-1 } \\
\text { CONTENTS OF TEN POLYPHENOLS IN LEAVES OF CHINESE HAWTHORN } \\
\text { (Crataegus. pinnatifida Bge. var. major NE Br) DURING DEVELOPMENT }\end{array}$} \\
\hline & 20-May & 17-Jun & 15-Jul & 12-Aug & 9-Sep & 7-Oct \\
\hline Chlorogenic acid & $0.33 \pm 0.03$ & $0.51 \pm 0.03$ & $0.52 \pm 0.03$ & $0.55 \pm 0.03$ & $0.57 \pm 0.03$ & $2.03 \pm 0.13$ \\
\hline Eucomic acid & $2.29 \pm 0.26$ & $2.47 \pm 0.41$ & $2.09 \pm 0.26$ & $2.81 \pm 0.35$ & $2.16 \pm 0.34$ & $2.54 \pm 0.18$ \\
\hline Procyanidin B2 & $0.30 \pm 0.05$ & $0.46 \pm 0.10$ & $0.76 \pm 0.12$ & $0.82 \pm 0.09$ & $0.90 \pm 0.01$ & $1.41 \pm 0.05$ \\
\hline Epicatechin & $0.21 \pm 0.02$ & $0.56 \pm 0.07$ & $0.63 \pm 0.04$ & $0.73 \pm 0.04$ & $0.64 \pm 0.03$ & $2.96 \pm 0.19$ \\
\hline Procyanidin C1 & $0.00 \pm 0$ & $0.32 \pm 0.09$ & $0.65 \pm 0.08$ & $0.75 \pm 0.04$ & $0.74 \pm 0.05$ & $0.97 \pm 0.04$ \\
\hline Vitexin-glucoside & $3.50 \pm 0.39$ & $4.11 \pm 0.57$ & $3.66 \pm 0.25$ & $4.33 \pm 0.42$ & $3.72 \pm 0.56$ & $3.39 \pm 0.43$ \\
\hline Vitexin-2"-rhamnoside & $5.22 \pm 0.52$ & $5.52 \pm 0.76$ & $4.66 \pm 0.37$ & $5.61 \pm 0.64$ & $4.86 \pm 0.70$ & $4.48 \pm 0.55$ \\
\hline Vitexin & $0.04 \pm 0.01$ & $0.09 \pm 0.05$ & $0.06 \pm 0.03$ & $0.08 \pm 0.03$ & $0.09 \pm 0.02$ & $0.16 \pm 0.01$ \\
\hline Hyperoside & $0.01 \pm 0.01$ & $0.25 \pm 0.04$ & $0.35 \pm 0.02$ & $0.32 \pm 0.02$ & $0.43 \pm 0.04$ & $2.22 \pm 0.20$ \\
\hline Isoquercitrin & $0.01 \pm 0.01$ & $0.06 \pm 0.07$ & $0.24 \pm 0.10$ & $0.46 \pm 0.05$ & $0.32 \pm 0.04$ & $1.32 \pm 0.10$ \\
\hline Total & 11.91 & 14.36 & 13.63 & 16.47 & 14.44 & 21.49 \\
\hline
\end{tabular}


Variation of phenolic contents in leaves of different leaf sequence: Fig 5 showed the comparison of the ten phenolic constituents in leaves of different leaf sequences. Among leaves of different leaf sequences, the variation of total content of the ten phenols was not significant, from $21.34 \mathrm{mg} / \mathrm{g}$ dry weight to $27.42 \mathrm{mg} / \mathrm{g}$ dry weight. Among these components, flavonoid was the highest one and was the main component in Chinese hawthorn leaves, especially the three $\mathrm{C}$-glycosylflavones. Their content in base and middle part of a twig both exceeded ten $\mathrm{mg} / \mathrm{g}$ dry weight and in all those eight leaves, the total amount of the three C-glycosylflavones all exceeded that of the two $\mathrm{O}$-glycosylflavones. There was another obvious trend: the rate of C-glycosylflavones to O-glycosylflavones $(\mathrm{C} / \mathrm{O})$ decreased as the leaf sequence increased. In the $1^{\text {st }}$ leaves on the base of twigs the rate of $\mathrm{C}$-glycosylflavones to $\mathrm{O}$-glycosylflavones $(\mathrm{C} / \mathrm{O})$ was 8.08 , whereas in the $4^{\text {th }}$ leaves on the middle of twigs it was 4.56 and in the $8^{\text {th }}$ leaves on the top of twigs it decreased to 1.83 . This trend has not been noticed before. This result showed that $\mathrm{C}$-glycosylflavones had its dominating role in base leaves which burgeoned out in spring and the rate of $\mathrm{O}$-glycosylflavones in leaves burgeoned later increased gradually. That would be one of the reasons of the increase of $\mathrm{O}$ glycosylflavones in autumn leaves. Besides, from the result shown in Fig. 5 it can be seen that the first leaves collected in autumn contents relatively a small amount of O-glycosylflavones and this was different from the almost undetectable O-glycosylflavones in young leaves shown in Fig. 3(B) and (D). This indicated that as the plant grew, the synthesis and accumulation of the O-glycosylflavones in the old leaves at the base of a twig had indeed increased and that would be another factor that leads to the increase of O-glycosylflavones in autumn leaves.

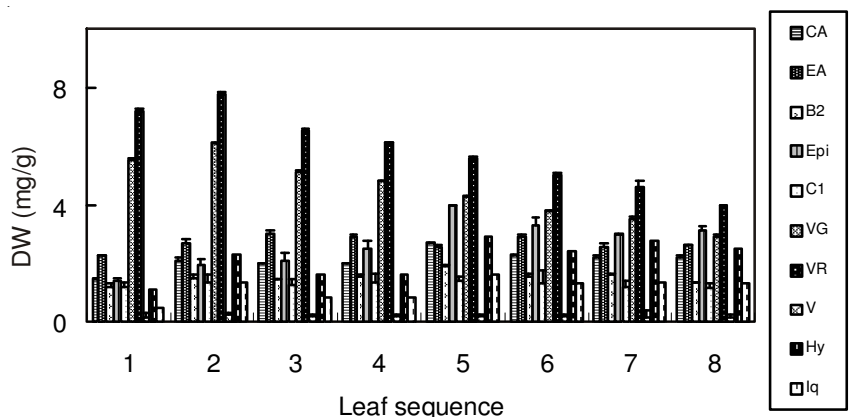

Fig. 5. Content of polyphenols in old leaves of Chinese Hawthorn (Crataegus. pinnatifida Bge. var. major $\mathrm{NE} \mathrm{Br)} \mathrm{with} \mathrm{Different} \mathrm{Leaf}$ Sequence. CA: chlorogenic acid, EA: eucomic acid, B2: procyanidin $\mathrm{B} 2$, Epi: epicatechin, $\mathrm{C} 1$ : procyanidin $\mathrm{C} 1$, VG: vitexin-glucoside, VR: vitexin-2"-rhamnoside, V: vitexin, Hy: hyperoside, IQ: isoquercitrin. Values are expressed as means \pm SD of triplication
The results showed that, as European hawthorn, Chinese hawthorn also contain high amount of phenolic components, but the composition and contents of these phenolic compounds were different between the two species of hawthorn. Different part of the twig and collecting time may affect the contents of phenolic components in the hawthorn leaves and thus affects the manufacture productions or even the efficacy and the quality of the drug. Autumn is exactly the time of fruit maturation, so the collection of the leaves, which contain high level of phenols, would have relatively insignificant impacts on fruit trees. Therefore it should be a very convenient resource of edible phenols.

\section{ACKNOWLEDGEMENTS}

This project was financially supported by the the National Natural Science Foundation of China (31071494) and Natural Science Foundation of Hebei Provience (C2011204093).

\section{REFERENCES}

1. W.T. Chang, J. Dao and Z.H. Shao, Am. J. Chin. Med., 33, 1 (2005).

2. H.P.T. Ammon and R. Kaul, Dtsch. Apoth. Ztg., 134, 33 (1994).

3. U. Svedström, H. Vuorela, R. Kostiainen, K. Huovinen, I. Laakso and R. Hiltunen, J. Chromatogr. A, 968, 53 (2002).

4. J. Wittig, I. Leipolz, E.U. Graefe, B. Jaki, D. Treutter and M. Veit, Arzneimittelforschung, 52, 89 (2002).

5. U. Svedström, H. Vuorela, R. Kostiainen, I. Laakso and R. Hiltunen, J. Chromatogr. A, 1112, 103 (2006).

6. China Pharmacopoeia Committee. Chinese Pharmacopoeia (I), Chemical Industry Press, Beijing, p. 576 (2005)

7. G.X. Hu, H.J. Yu, Y.D. Shao and L.J. Fang, Chin. Trad. Herbal Drugs, 33, 905 (2002).

8. H.W. Li, S.Q. Zhang, J.P. Dou and Y.S. Xi, Lishizhen Med. Mater. Med. Res., 18, 773 ( 2007).

9. R.H. Liu, A.L. Jiang, L.L. Bian, S. Feng, Z.L. Ma and B.Y. Yu. J. Chin. Med. Mater., 30, 285 (2007).

10. W. Heller and C. Tamm, Helv. Chim. Acta, 57, 1776 (1974).

11. M. Ishii, Y. Shoyama, S. Uemoto, I. Nishioka and K. Fujieda, Gakugei Zasshi-Kyushu Daigaku Nogakubu, 31, 99 (1976).

12. X.B. Ding, Y.Q. Jiang, Y. Zhong and C.X. Zuo, China J. Chin. Mater. Med., 15, 39 (1990).

13. J.Q. Jiang, W. Ye, Z. Chen, F. Lou and Z. Min, J. Chin. Pharm. Sci., 11, 1 (2002).

14. A. Mun'im, O. Negishi and T. Ozawa, Biosci. Biotechnol. Biochem., 67, 410 (2003).

15. W.M. Liu, G.H. Chen and T. Cui, J. Chromatogr. Sci., 41, 87 (2003).

16. T. Cui, J.Z. Li, H. Kayahara, L. Ma, L.X. Wu and K. Nakamura, J. Agric. Food Chem., 54, 4574 (2006).

17. T. Cui, K. Nakamura, S. Tian, H. Kayahara and Y.L. Tian, Biosci. Biotechnol. Biochem., 70, 2948 ( 2006).

18. G.Y. Gao, X.Q. Qin, M. Li and Q.X. Feng, Nat. Prod. Res. Devel., 6, 27 (1994).

19. J. Su, W.D. Zhang, Y. Zhou, J. Zhou and Z.B. Gu, China J. Chin. Mater. Med., 29, 525 (2004).

20. A. Rehwad, B. Meier and O. Sticher, J. Chromatogr. A, 677, 25 (1994).

21. R.P. Shi, Q.Y. Zhou and X.J. Chen, Chin. Pharm., 11, 55 (2002). 